\title{
BMJ Open Impact of a Pozzi tenaculum forceps use on the success rate of intrauterine insemination (IUI) : protocol for a randomised control trial
}

\author{
Stephanie Dufour (1) , ${ }^{1}$ Mathieu Viau, ${ }^{2}$ Maxime Côté, ${ }^{1}$ Jessica Lefebvre, ${ }^{3}$ \\ Sarah Maheux-Lacroix, ${ }^{3}$ Marie-Ėve Bergeron ${ }^{3}$
}

To cite: Dufour S, Viau M, Côté M, et al. Impact of a Pozzi tenaculum forceps use on the success rate of intrauterine insemination (IUI) : protocol for a randomised control trial. BMJ Open 2021;11:e043230. doi:10.1136/ bmjopen-2020-043230

- Prepublication history is published online only. To view please visit the journal online (http://dx.doi.org/10.1136/ bmjopen-2020-043230)

Received 30 July 2020 Revised 05 February 2021 Accepted 19 February 2021

Check for updates

(C) Author(s) (or their employer(s)) 2021. Re-use permitted under CC BY-NC. No commercial re-use. See rights and permissions. Published by BMJ.

${ }^{1}$ Obstetrics and Gynecology, $\mathrm{CHU}$ de Québec-Université Laval, Quebec, Quebec, Canada ${ }^{2}$ Obstetrics and Gynecology, Sherbrooke Hospital, Sherbrooke, Quebec, Canada ${ }^{3}$ Gynecology and Reproductive Endocrinology, CHU de Québec - Université Laval, Québec City, Québec, Canada

Correspondence to Dr Marie-Ève Bergeron, Gynecology and Reproductive Endocrinology, CHU de Québec - Université Laval, Québec,

Québec, Canada;

meb@mebergeron.com

\section{ABSTRACT}

Introduction A therapeutic option frequently proposed for infertility is to proceed first with intrauterine insemination (IUI) cycles. Little is known on the effect of uterine contractions on IUI success rates. We postulated that uterine contractions may help with sperm migration in the women's genital tract. Our objective is to compare the efficacy of IUI when performed with and without a tenaculum forceps on the cervix.

Methods and analysis We are conducting a randomised controlled trial with women aged 18-40 years old, diagnosed with primary or secondary infertility due to endometriosis, mild to moderate male factor, unexplained infertility or ovulatory dysfunction, receiving IUI treatments. On the day of their IUI, women will receive standard care treatment plus or minus the planned use of a Pozzi tenaculum forceps on the cervix. Each of the 800 women will be randomised only once for their first three IUI. They will be randomised in one of the following sequences: tenaculum - no tenaculum - tenaculum or, no tenaculum —-tenaculum — no tenaculum. We will compare the live birth rate (primary outcome) and the clinical pregnancy rate (secondary outcome) after IUI treatment. We also plan on assessing global satisfaction of women, pain levels felt during IUI and discrepancies in primary and secondary outcomes in interventions performed by attendings compared with trainees.

Ethics and dissemination This research project was approved by the Centre Hospitalier Universitaire de Québec's ethics review board (no 2018-4084). Pozzi tenaculum application is associated with mild pain, estimated at 33 on a Visual Analogue Scale ranging from 0 to 100 . We plan on publishing an article to present our findings in a peer-reviewed journal as well as presenting the research abstract at conferences.

Trial registration number NCT03435809.

\section{INTRODUCTION}

A therapeutic option frequently proposed for infertile couples is to proceed first with intrauterine insemination (IUI) cycles with ovarian stimulation since some couples will successfully conceive without requiring in vitro fertilisation (IVF) treatments. ${ }^{1}$ Advantages of this minimally invasive technique
Strengths and limitations of this study

- This study could benefit society as a whole by reducing infertility treatment cost and increasing treatment success; intrauterine insemination could gain interest and is significantly less costly than in vitro fertilisation.

- This trial will compare live birth rates as its primary outcome; it is the main outcome sought by patients treated for infertility.

- This study takes into account the women's perspective on the treatment and its acceptability.

- This study is a single-blinded study which could lead to potential bias in statistical analysis.

include a fair pregnancy rate increase, compared with the low complication rate, its low cost and its availability. ${ }^{12}$ Despite these advantages, this less invasive treatment still as a lower pregnancy compared with IVF. ${ }^{1}$ Thus, any improvement in the widely used IUI technique would benefit couples. Moreover, there is scarce literature on the effect of uterine contractions during IUI on its success rate. Our literature review on the use of tenaculum forceps during IUI revealed two studies on this subject. Only one randomised controlled trial by Balci et al reported an increased pregnancy rate for women on whom a tenaculum forceps was put on the cervix during the IUI. ${ }^{3}$ They suggested that this increase in success rate might be secondary to increased contractions mimicking intercourse and helping with sperm migration in women's upper genital tract.

The second study, a retrospective analysis by Park et al, compared success rates of IUI during difficult procedures where tenaculum had to be used to those where it was not required. ${ }^{4} \mathrm{~A}$ trend for clinical pregnancy rate toward not using a tenaculum was not statistically significant. The study design in 
this case is a major weakness; women for whom a tenaculum was required to pass the catheter through the cervix could have had a worse fertility prognosis related to past surgical or gynaecological medical history.

Our literature review shows lack of prospective research without selection bias. An adequate method to validate the technique's benefit would be a randomised controlled study eliminating most of the prognostic confounding factors.

We designed this randomised controlled trial to compare live birth rates (primary outcome) following IUI performed with and without the placement of a Pozzi tenaculum forceps on the cervix. Secondary outcomes will include clinical pregnancy rate, global satisfaction of women, pain levels felt by women during IUI and discrepancies in primary and secondary outcomes in cycles performed by attendings compared with trainees.

\section{METHODS AND ANALYSIS}

\section{Study setting}

This study is conducted at the fertility clinic of the Centre Hospitalier Universitaire (CHU) de Québec. It is a secondary infertility care centre were approximately 2000 IUI cycles are performed each year. This protocol is written in accordance with the Standard Protocol Items: Recommendations for Interventional Trials. Any changes from the original protocol will be reported in the final article.

\section{Study design}

Women aged 18-40years old, diagnosed with primary or secondary infertility due to endometriosis, mild to moderate male factor, unexplained infertility or ovulatory dysfunction, having IUI treatments with the use of oral medication for controlled ovulation induction or stimulation will be invited to participate in the study.

\section{Eligibility criteria}

Eligibility criterions are found in box $1 .^{56}$

\section{Recruitment}

Participants will be managed by gynaecologists from the department of gynaecology, reproductive endocrinology and infertility from the CHU de Québec - Université Laval. Once couples and their treating physician (gynaecologist) agree on IUI treatments, those who are eligible will be offered to participate in our study. The gynaecologist will then explain the research project and will obtain consent from the couples during the consent appointment already taking place before IUI are started.

During this consent meeting, an information sheet will be handed to participants and their questions will be answered. A distinct consent form for IUI will also be signed by the couples. Another study consent form, clearly identified, will be presented to couples interested in enrolling in the study and will be signed at that moment. As the partner's semen is used for the IUI, they

\section{Box 1 Eligibility criteria}

\section{Inclusion criteria}

1. Women aged $\geq 18$ and $\leq 40$ years.

2. Primary or secondary infertility $\geq 12$ months.

3. Known medical indication for intrauterine insemination (IUI)

A. Endometriosis.

B. Mild to moderate male factor (total motile count $\geq 5 \mathrm{M}$ in initial sperm assessment).

C. Ovulatory dysfunction.

D. Unexplained infertility.

E. Mixed infertility of above known indication.

4. First fertility treatment or first treatment since last pregnancy.

5. Ovulation induction or stimulation with oral medication.

6. Being able to speak and understand French.

\section{Exclusion criteria}

1. Bilateral tubal occlusion.

2. Active pelvic infection.

3. $<1 \times 10^{6}$ mobile sperm on semen analysis.

4. Donor sperm for IUI.

will also receive information and sign the consent form if they agree to participate.

Before the first IUI cycle, each participant's electronic file will be reviewed by one of the main investigators to confirm or infirm eligibility criteria are met, and they will complete data collection with sociodemographic information. Baseline data to be collected include : women and partners' age, ethnicity, women's weight $(\mathrm{kg})$ and height (m), smoking habit, weekly alcohol consumption, illicit drug use, primary versus secondary infertility, obstetrical history expressed by the gravida/para/abortus (GPA) nomenclature, cycle duration (days), infertility duration (months), infertility etiology, antral follicle count (AFC), antimullerian hormone level (AMH), follicle stimulating hormone (FSH) level and tubal permeability. ${ }^{6-9}$

\section{Randomisation}

Randomisation will proceed with computer-generated randomisation of sealed opaque envelopes in block of 4. The statistics department at the Centre de Recherche du CHU de Québec will carry out the randomisation process. It will be a 1:1 ratio between the intervention and control groups. ${ }^{10}$ Each woman will be randomised only once for their first three ${ }^{3}$ IUI. They will be randomised in one of the following sequences: tenaculum-no tenaculum-tenaculum or, no tenaculum-tenaculum-no tenaculum .

\section{Blinding}

Women will be blinded to their group allocation. The attending physician or the trainee doing the IUI will not be blinded to the group allocation; they must be aware of the need to use a tenaculum or not.

\section{Intervention}

Management of each IUI cycle, medication, ultrasound and ovulation induction will proceed as usual 
for women. Once the initial investigations are done, an oral controlled ovarian stimulation is started on the first cycle if medically indicated. At mid-cycle, patients undergo a transvaginal follicular scan. This ultrasound's goal is to detect ovulation and plan the IUI. If no follicle is detected, the transvaginal ultrasound scan is repeated later. If there is no luteinizing hormone (LH) peak detected on urinary ovulation test, an ovulation trigger with a human chorionic gonadotropin (hCG) based medication will be administered to patients depending on patient's age, number and size of follicles detected. Subcutaneous hCG or intramuscular hCG can be used. A cycle can be cancelled if there is no ovulation or if there is ovarian hyperstimulation.

For those who are randomised to the intervention group, a Pozzi tenaculum forceps will be applied and clamped horizontally to the anterior cervical labrum with a single click and mild traction will be applied in order to decrease the cervico-uterine angle. When no tenaculum will be applied, motion will be mimicked without actual tissue prehension. Women randomised to the control group will only have a tenaculum forceps applied in the rare cases where the IUI will not be feasible otherwise by the gynaecologist. Indeed, it is sometimes necessary, when the cervical anatomy does not allow for easy pass of the catheter, for a Pozzi tenaculum forceps to be used in order to insert the insemination catheter properly in the uterine cavity. In that case, tenaculum use will be recorded in the file. No cervical insemination will be allowed. Insemination will be performed with flexible catheters and care will be taken to avoid touching the uterine fundus with the catheter tip. There are multiple catheters available in the gynaecology clinic, namely catheters TheCurve (Cooper Surgical, Berlin), Shapeable IUI Catheter (Thomas Medical, Indianapolis) and Minispace (Laboratoire C.C.D., Paris). After the intervention, women will be instructed to lay flat for 10 minutes after which they will go home until next appointment.

Intercourse will be allowed following IUI treatments. Women will be instructed to perform a urinary pregnancy test at home 2 weeks after the IUI, which will be provided to them. If the test is positive, women will be scheduled for a transvaginal ultrasound scan on the 7 th week of amenorrhea to confirm proper intrauterine pregnancy evolution. If intrauterine pregnancy is confirmed, women will be referred to a physician for obstetrical follow-up.

In case of treatment failure, women will go on with ovulation induction or stimulation for the next cycle as previously prescribed by the gynaecologist at the midcycle ultrasound and if indicated. Dosages of ovarian stimulation medication may be modified, at physician's discretion, in order to optimise response to treatment according to the usual indications (ovarian hyperstimulation, side effects, poor response, etc) Women will be followed for three cycles and, if need be, can receive subsequent IUI treatments outside research protocol.

\section{Outcomes}

\section{Primary outcome measure}

Each medical visit will be recorded in an electronic file system (Medifirst) were data will be collected from. For each cycle, data on stimulation medication and doses, number of follicles $\geq 14 \mathrm{~mm}$, endometrial thickness, day of the menstrual cycle, ovulation induction injection use and concentration of sperm after preparation results will be collected.

If a pregnancy is diagnosed clinically at the ultrasound visit, a second questionnaire will be handed to women. They will be asked to fill in the questionnaire on pregnancy outcomes after delivery and to send it back to the investigators. On this form, women will report if they had a singleton or multiple pregnancy and the outcome. A follow-up phone call will be done, or a written follow-up will be sent if no questionnaire is received 1 month passed the due date. Collected data will be used to assess live birth rate. In the event of an adverse outcome (spontaneous abortion, fetal demise, neonatal death), women will be directed to appropriate resources.

\section{Secondary outcome measures}

We will also compare clinical pregnancy rate (positive urine pregnancy test or biochemical) after IUI treatment. At each insemination visit, we will record the woman's satisfaction regarding the treatment (self-reported, yes/ no questions) and pain felt during the procedure on a Visual Analogue Scale (VAS) from 0 to 10.

Discrepancies in primary and secondary outcomes in interventions performed by attendings compared with trainees will also be assessed.

\section{Sample size}

The total sample of 400 cases at the time of the interim analysis provides $63 \%$ power of Fisher's exact test to detect $17.7 \%$ of pregnancy following the use of Pozzi forceps compared with $10.7 \%$ of pregnancy without the use of forceps. The same estimation with 800 cases provides $80 \%$ of the power. All power was estimated using GPower V.3.1 with a significance level of 0.05 .

To estimate the power of a generalised estimating equation (GEE) logistic model that takes into account 1-3 cycles of inseminations, a sample of 400 simulated cases was created with a $17.7 \%$ pregnancy prevalence following the use of Pozzi forceps and $10.7 \%$ prevalence of pregnancy without the use of Pozzi forceps. A total of 1000 samples were resampled using bootstrap unrestricted random sampling from the simulated database. The power of the GEE logistics model was $43 \%$ for a total sample of 400 cases. The same procedure for a total sample of 800 cases provides $80 \%$ power for GEE logistic regression model. All estimation was done using SAS V.9.4.

Finally, this sample size estimation $(\geq 180$ cases by group) is in agreement with the recommendations of Rochon for GEE logistic regression with three repeated measurements (new). ${ }^{10}$ 


\section{Statistical analysis}

We will compare the live birth rate difference between the two groups using a generalised linear mixed model with correlated binary outcomes for three-time repeated measures. ${ }^{11} \mathrm{~A} p<0.05$ is considered statistically significant. Probability of live birth with each IUI cycle will be determined with an OR including a 95\% CI.

A per-protocol and intention-to-treat analysis will be done. We will proceed with an interim analysis when 400 women are recruited and have completed their treatments. The interim analysis has a power of $63 \%$ to detect an equivalent deleterious effect.

Statistical analysis to compare participants' characteristics (age, body mass index, smoking status, alcohol consumption, illicit drug use, infertility primary vs secondary, GPA, duration of infertility, aetiology of infertility, number of IUI, AFC, FSH, mature follicles on each cycle) and partners' characteristics (age, semen analysis) between couples being randomised to the intervention compared with the control group. If any imbalance between the groups is noticed, a logistic regression analysis will be used.

\section{Patient and public involvement}

No patient involved

A summary of schedule of enrolment, allocation, intervention and assessments can be found in figure 1 .

\section{Ethics and dissemination}

Participation to the study will be under free-will with proper informed consent. Women will be able to withdraw from the study at any point in time. Collected data are confidential, non-nominal and stored in a secured electronic file. It will only be accessible to the main

\begin{tabular}{|c|c|c|c|c|c|c|c|}
\hline \multirow[b]{2}{*}{ TIMEPOINT } & \multirow{2}{*}{$\begin{array}{c}\text { Enrolment } \\
0\end{array}$} & \multirow{2}{*}{$\begin{array}{c}\text { Allocation } \\
0\end{array}$} & \multicolumn{4}{|c|}{ Post-allocation } & \multirow{2}{*}{$\begin{array}{c}\begin{array}{c}\text { Close- } \\
\text { out }\end{array} \\
t_{x} \\
\end{array}$} \\
\hline & & & $\begin{array}{l}\text { IUI } \\
\# 1\end{array}$ & $\begin{array}{l}I U 1 \\
\# 2\end{array}$ & $\begin{array}{l}I U I \\
\# 3\end{array}$ & $\begin{array}{l}E D D^{1}+ \\
1 \text { month }\end{array}$ & \\
\hline \\
\hline Eligibility screen & $\mathrm{x}$ & & & & & & \\
\hline Informed consent & $x$ & & & & & & \\
\hline & & $\mathrm{x}$ & & & & & \\
\hline \\
\hline $\begin{array}{r}\text { No tenaculum \#1 } \\
\text { Tenaculum \#2 } \\
\text { No tenaculum \#3 } \\
\text { sequence }\end{array}$ & & & $\mathrm{x}$ & $(X)^{2}$ & $(\mathrm{X})$ & $+/-^{3}$ & \\
\hline $\begin{array}{r}\text { Tenaculum \#1 } \\
\text { No tenaculum \#2 } \\
\text { Tenaculum \#3 } \\
\text { sequence } \\
\end{array}$ & & & $\mathrm{x}$ & (X) & (X) & + +- & \\
\hline ASSESSMENTS: & & & & & & & \\
\hline $\begin{array}{r}\text { Women age } \\
\text { Partnerage } \\
\text { Ethnicity } \\
\text { Women's weight (kg) } \\
\text { Women's height (m) } \\
\text { Smoking habit } \\
\text { Alcohol consumption } \\
\text { Illicit drug use } \\
\text { 1ry or 2ry infertility } \\
\text { Obstetrical history (GPA) } \\
\text { Cycle duration } \\
\text { Infertility duration } \\
\text { Infertility etiology } \\
A F C \\
\text { AMH } \\
\text { FSH levels } \\
\text { Tubal permeability }\end{array}$ & $\mathrm{x}$ & $\mathrm{x}$ & & & & & \\
\hline $\begin{array}{r}\text { Type oral stimulation medication } \\
\text { and dose } \\
\text { Number of follicles }(\geq 14 \mathrm{~mm}) \\
\text { Endometrial thickness } \\
\text { Day of the menstrual cycle } \\
\text { Concentration of sperm after } \\
\text { preparation } \\
\text { Ovulation induction agent }\end{array}$ & & & $\mathrm{x}$ & $\mathrm{x}$ & $\mathrm{x}$ & & \\
\hline $\begin{array}{r}\text { Patient questionnaire } \\
\bullet \quad \text { Global satisfaction } \\
\bullet \quad \text { VAS } \\
\text { Urine pregnancy test }\end{array}$ & & & $\mathrm{x}$ & $\mathrm{x}$ & $\mathrm{x}$ & & \\
\hline Pregnancy outcome & & & & & & $x$ & $x$ \\
\hline
\end{tabular}

Figure 1 Summary of schedule of enrolment, interventions and assessments. 1 EDD, estimated due date; 2, If pregnancy test negative in previous cycle; 3 , If positive pregnancy test obtained in any cycle. AFC, antral follicle count; $\mathrm{AMH}$, antimullerian hormone level; IUI, intrauterine insemination; VAS, Visual Analogue Scale. 
investigators and the statistical department at the Centre de recherche clinique du CHU de Québec helping out for the statistical analysis. Data will be kept for 10 years by the main investigator, then deleted.

This research project was approved by the CHU de Québec's ethics committee and review board (no 2018-4084) before recruitment started. Annual renewal of the approval will be required by the ethics committee.

Possible inconvenient resulting from participation in the study includes time for protocol explanation and consent. Also, the Pozzi tenaculum application is associated with mild pain, estimated at 33 on a VAS ranging from 0 to $100 .{ }^{12}$ In the gynaecology practice, no local anaesthetic is regularly used in IUI with or without a tenaculum applied. Women will be allowed to take medication before the procedure (eg, acetaminophen) in the study frame. Apart from the tenaculum use, fertility treatments with IUI will proceed as usual and are under the treating physician's responsibility.

Recruitment of participants began in June 2019. We anticipate that recruitment will be completed by June 2022. Additional time for data collection will be required as three cycles of IIU are included and pregnancy outcomes are collected. We are planning to present the findings in local and national meetings and publish the results in a peer-reviewed journal. Other opportunities to share our results may be considered.

In return for previous named inconvenient, couples will participate in the improvement of fertility treatments which can have a positive impact on them and can benefit society as a whole by reducing treatments' cost and increasing treatments' success. Potential profit resides in the fact that pregnancy tests will be freely handed to women.

Contributors MV and SD under the supervision of M-ĖB, developed the objectives of the study and established their relevance in relation to the current literature. MV developed the methodology and drafted the first version of the manuscript with significant input from all other authors. SD, SM-L, M-ĖB, JL and MC ensured the methodological quality of the study and MC, SD and M-ËB led integration into the clinical setting. All the authors have read and approved the final version of the manuscript.

Funding This work is and will be supported by the Fonds de recherche du service de gynécologie reproduction du Centre Hospitalier de l'Université Laval - CHU de Québec.

Competing interests None declared.

Patient and public involvement Patients and/or the public were not involved in the design, or conduct, or reporting, or dissemination plans of this research.
Patient consent for publication Not required.

Provenance and peer review Not commissioned; externally peer reviewed.

Open access This is an open access article distributed in accordance with the Creative Commons Attribution Non Commercial (CC BY-NC 4.0) license, which permits others to distribute, remix, adapt, build upon this work non-commercially, and license their derivative works on different terms, provided the original work is properly cited, appropriate credit is given, any changes made indicated, and the use is non-commercial. See: http://creativecommons.org/licenses/by-nc/4.0/.

ORCID iD

Stephanie Dufour http://orcid.org/0000-0003-2237-9843

\section{REFERENCES}

1 The ESHRE Capri Workshop Group. Intrauterine insemination. Hum Reprod Update 2009;15:277 https://academic.oup.com/humupd/ article-lookup/doi/ doi:10.1093/humupd/dmp003

2 Dinelli L, Courbière B, Achard V, et al. Prognosis factors of pregnancy after intrauterine insemination with the husband's sperm: conclusions of an analysis of 2,019 cycles. Fertil Steril 2014;101:994-1000 https://linkinghub.elsevier.com/retrieve/pii/S0015028214000399 doi:10.1016/j.fertnstert.2014.01.009

3 Balci O, Acar A, Colakoglu MC. Does tenaculum application to the cervix during intrauterine insemination affect pregnancy rates? Acta Obstet Gynecol Scand 2009;88:1053-6 http://doi.wiley.com/ doi:10.1080/00016340903118000

4 Park KE, Ku S-Y, Kim H, et al. The use of tenaculum during intrauterine insemination may not affect the pregnancy outcome. Yonsei Med J 2010;51:949 https://eymj.org/DOlx.php?id= doi:10.3349/ymj.2010.51.6.949

5 Collège des médecins du Québec. Les activités de procréation médicalement assistée - démarche clinique et thérapeutique. CMQ 2015:1-138.

6 Cooper TG, Noonan E, von Eckardstein S, et al. World Health organization reference values for human semen characteristics*ł. Hum Reprod Update 2010;16:231-45 http://academic.oup.com/ humupd/article/16/3/231/639175/World-Health-Organizationreference-values-for doi:10.1093/humupd/dmp048

7 Demir B, Dilbaz B, Cinar O, et al. Factors affecting pregnancy outcome of intrauterine insemination cycles in couples with favourable female characteristics. J Obstet Gynaecol 2011;31:420-3 http://www. tandfonline.com/doi/full/ doi:10.3109/01443615.2011.569780

8 Aydin $\mathrm{Y}$, Hassa H, Oge T, et al. Factors predictive of clinical pregnancy in the first intrauterine insemination cycle of 306 couples with favourable female patient characteristics. Hum Fertil 2013;16:286-90 http://www.tandfonline.com/doi/full/ doi:10.3109/14 647273.2013.841328

9 Broekmans FJM, de Ziegler D, Howles CM, et al. The antral follicle count: practical recommendations for better standardization. Fertil Steril 2010;94:1044-51 https://linkinghub.elsevier.com/retrieve/pii/ S0015028209010085 doi:10.1016/j.fertnstert.2009.04.040

10 Rochon J. Application of Gee procedures for sample size calculations in repeated measures experiments. Stat Med 1998; 17:1643-58.

11 Dang Q, Mazumdar S, Houck PR. Sample size and power calculations based on generalized linear mixed models with correlated binary outcomes. Comput Methods Programs Biomed 2008;91:122-7 https://linkinghub.elsevier.com/retrieve/pii/ S0169260708000758 doi:10.1016/j.cmpb.2008.03.001

12 Hoffman BLS, Schorge JO, Schaffer JI. Williams gynecology. 2nd edn. Dallas: McGraw-Hill, 2008. 